\title{
Research of Enterprise Knowledge Absorption Strategy Based on Big Data Platform
}

\author{
Jianchun Yang ${ }^{1,2}$ Ruo $\mathrm{Shi}^{3}$ \\ 1School of Business Administration, Guizhou University of Finance and Economics, \\ Guiyang 550025, China \\ 2Research Institute of Guizhou Industrial Economics and Management, Guizhou University of Finance \\ and Economics, Guiyang 550025, China \\ 3School of Finance, Guizhou University of Finance and Economics, Guiyang 550025, China

\section{基于大数据平台的企业知识吸收策略研究 杨建春 ${ }^{1,2}$ 施若 $^{3}$ \\ 1. 贵州财经大学工商学院, 贵阳 550025 , 中国 2. 贵州财经大学贵州工业经济与管理研究院, 贵阳 550025, 中国 \\ 3. 贵州财经大学金融学院, 贵阳 550025, 中国}

\begin{abstract}
With the coming of the Big Data period, it is crucial for business to deal with customer data. First, we analyze the characteristics of customer dada: volume and diversity. Then, we disucss the challange business faced with in organization management practice. Bsed on this, we put forward three strategies of improving knowledge absorptive capability and show how organization leaders can build the absorptive capability necessary to extract business value from customer data.
\end{abstract}

Keywords: Big Data ; Customer data ; Knowledge absorptive capability

\section{摘要}

随着大数据时代的来临, 企业如何应对来 自顾客的数据显得至关重要。首先分析了顾客 数据具有巨量和多样性的特征, 然后探讨了这 两类特征对企业管理实践带来的挑战。在此基 础上, 提出了提高企业知识吸收能力的三大策 略, 展示了企业如何培养必要的知识吸收能 力, 从顾客数据中获取商业价值。

关键字: 大数据; 顾客数据; 知识吸收能力
1. 引言

随着大数据时代的来临, 企业如何挖掘各 种利益相关者的创新能力、知识和经验, 显得 至关重要。例如, 黄金生产商 GoldCorp 构建 了一个公众可以使用的地理数据库, 任何人只 要告诉公司在哪儿能找到金子都可以获得奖 励。这一行动的结果是 GoldCorp 的黄金产量 从 53000 盎司一年增加到 504000 盎司一年, 成本从 360 美元每盎司降至 59 美元每盎司。 因此, GoldCorp 公司的市值也从 1 亿美元增 至 90 亿美元。以上案例说明企业可以成功从 以顾客为主的外部数据中获取商业价值 ${ }^{[1]}$ 。

成功的顾客关系管理平台很容易吸引成 千上万的活跃顾客, 这些顾客创造了大量的的 数据。企业面对这些大数据时, 往往不知所措, 导致耗费大量时间挖掘其中的商业价值, 并且 占用了大量的资源, 付出了很高的代价。特别 是当企业缺乏理解和使用众包数据的能力, 会 使得付出的努力超出了带来的收益, 反而增加 了企业的负担 ${ }^{[2]}$ 。因此, 如何提高企业的知识 吸收能力, 对于企业从顾客数据中获取商业价 值至关重要。

\section{2 顾客数据的特征}

大数据环境下, 即使建立了一个如 BetaCorp IdeaZone 成功的顾客关系管理平 
Risk Analysis and Crisis Response in Big Data Era (RAC-16)

台, 如何有效挖掘顾客数据仍然是项挑战。这 是因为顾客数据的特征, 不利于企业评估、传 输和吸收这些数据。顾客数据具有以下两大特 征: 巨量和多样性（如图 1 所示)。

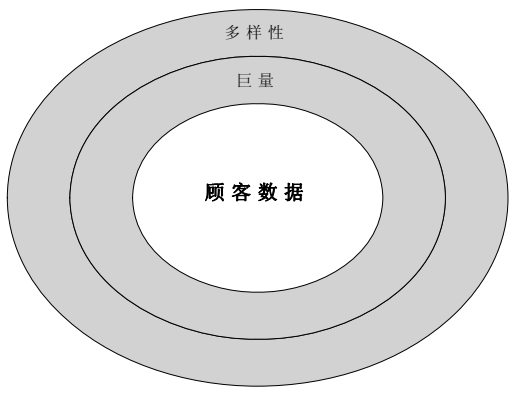

图 1 顾客数据的特征

\section{1 巨量}

顾客数据的第一个特征: 巨量。顾客关系 管理平台可以收集到源自顾客的大量数据。这 些数据分为三种类型: 1) 解决方案: 创意或 商业计划; 2) 元数据: 包括评估、评论或标 签; 3 ) 顾客自身数据: 个人特征、活动、偏 好等。顾客数据的巨量, 主要是由于数据产生 的速度导致的。例如 AlphaCorp 平台在其运行 的第一周内, 就产生了超过 8000 个贡献。

\section{1 多样性}

顾客数据的第二个特征: 多样性。为了促 进顾客最大程度的参与, 企业对潜在的参与者 不施加任何格式和结构限制。正是因为如此, 顾客数据可能缺乏聚焦。对于同一任务来说, 顾客数据可以是文本描述、图形可视化, 也可 以是开发原型, 它们在格式上显著不同。此外, 顾客关系管理平台吸引有着不同背景的参与 者, 这些参与者对同一任务, 提出不同的解决 方案。这些解决方案有很大的不同, 大多数解 决方案是低价值的, 这些低质量的解决方案往 往是高度模糊和不具体的。

\section{3 顾客数据特征给知识吸收过程带来的挑战}

本文先把大数据环境下的知识吸收过程 分为数据评价、数据传输和数据同化三个阶段 (如图 2 所示)。然后, 再来论述顾客数据特
征给知识吸收过程每个阶段带来的影响。

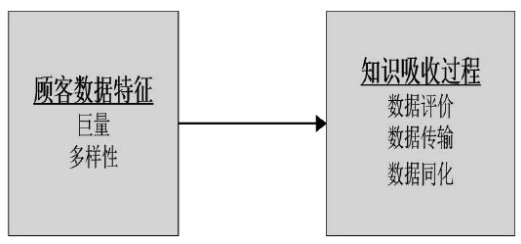

图 2 知识吸收过程面临的挑战

\section{1 数据评价}

对于企业来说, 数据巨量和多样性使得解 决方案更难以评估。巨量的数据使得人工不可 能评价所有的数据, 数据的多样性也阻碍了评 估工作的自动化。另外, 企业也可能缺乏足够 的先验知识来评估这些数据。因此, 企业采用 各种评价机制, 例如咨询顾客如何对解决方案 进行评级。然而评价机制的设计和使用是具有 高度挑战性的。例如, 设计不合理评估量表会 导致接近随机的结果。此外, 企业很多时候并 没有意识到, 如果选用顾客来尝试解决方案的 评估, 同样是非常耗时的。通常结果是顾客仅 仅只评估了一小部分解决方案, 而很多解决方 案没有被评估。尤其是当评价指标被顾客误用 时，情况会更加槽糕。

\section{2 数据传输}

在数据传输阶段, 首先要识别和选择合适 的员工。业务部门负责消化数据并实现创意, 这是顾客数据的吸收过程中重要的一个阶段 ${ }^{[3]}$ 。不合适的接受者可能不理解数据并忽略数 据。由于数据的多样性, 来自顾客平台的数据 需要涉及不同的业务单位。因此, 为每个可行 的解决方案找到合适的雇员是具有挑战性的。

\section{3 数据同化}

顾客数据的同化过程是将顾客数据转换 成有价值信息的过程。企业通过结合数据与现 有的公司的知识来执行方案。例如, GammaCorp 选择实施的每个解决方案都会经历标准资源 分配过程，涉及技术可行性分析、经济可行性 分析和战略协调性 ${ }^{[4]}$ 。然而, 考虑到顾客数据 的特征, 同化过程是艰巨而漫长的。选择的解 决方案和相关数据要整合和修正, 这样解决方 
Risk Analysis and Crisis Response in Big Data Era (RAC-16)

案就可以被评估以应对对企业内部约束, 如战 略和资源约束。

\section{4 提高企业知识吸收能力的三大策略}

企业需要提高知识吸收能力, 把顾客数据 转化为知识和商业价值。因此, 知识吸收能力 决定了企业对顾客数据的评价、传输和吸收的 过程。提高顾客数据的吸收能力, 应对来自顾 客数据特征的种种挑战, 企业需要培养三大策 略: 平台设计、过滤设计和建立社区 (如图 3 所示)。

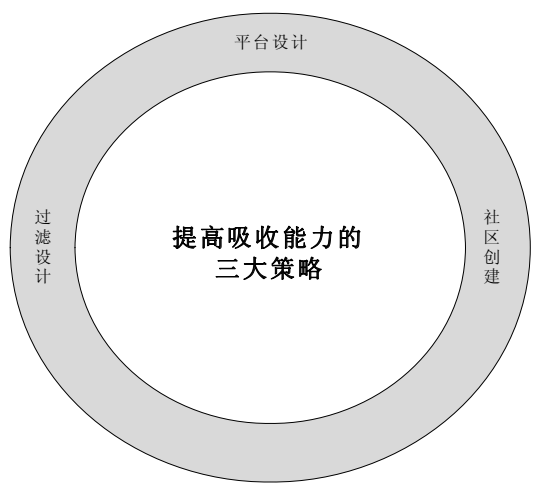

图 3 提高吸收能力的三大策略

\section{1 平台设计}

顾客平台构建了参与者如何给予贡献、如 何与其他参与者交互。平台制定了一定的标准 化流程, 包括定义顾客数据的结构、格式和质 量。因此, 一个设计良好的顾客平台, 可以获 得企业的支持和高度认可, 提高顾客数据的价 值, 便于顾客数据的传输和吸收。例如, AlphaCorp 要求其顾客参与者为改善其产品提 供新思路, 通过要求参与者提供需求和解决方 案信息, 极大地提高了解决方案的质量。

需求信息描述要求以及期望得到的结果, 解决方案信息描述如何解决需求, 或者如何解 决企业中存在的问题。AlphaCorp 的顾客平台 包括 “问题描述” 和 “解决方案”。通过输入 需求和解决方案信息, 顾客参与者不仅思考的 是问题本身, 更重要的思考如何解决问题。这 种分开有助于顾客参与者给予不同的贡献。企 业员工主要感兴趣的是, 企业中存在的问题和 客户需求如何有效的满足。因此, 促进顾客参
与者之间的合作对于提高贡献质量也很重要。 在数据池中, 所有的顾客参与者都可以看到其 他顾客参与者的贡献, 并且参与者可以探索、 评论和编辑贡献。例如维基百科、AlphaCorp 和 BetaCorp, 通过连接潜在的合作者支持协 作, 并对不同方案的优点引起激烈的讨论。

同样, BetaCorp 使用网络会议加强顾客 参与者之间的合作。例如, BetaCorp 的平台 允许顾客参与者举行头脑风暴会议。这些方法 都可以围绕某单一贡献建立讨论组和团队。基 于合作的顾客参与提高了顾客数据的质量和 可理解性, 便于顾客数据评价、传播和同化。

最后, 顾客平台必须激励顾客参与者。 AlphaCorp 的平台不仅激励合作, 追求最好的 解决方案, 也激励参与者之间的竞争。因此, 参与者经常提交额外的方案, 提高他们的贡 献, 并提高他们的声誉和价值。另外一个有效 的激励机制, 包括游戏化机制, 例如评级和积 分系统 ${ }^{[5]}$ 。BetaCorp 给每一个参与活动奖励积 分, 分数排名显示顾客参与者的活跃程度。 BetaCorp 使用这些方法, 都可以促进参与者 参与评论和评估, 现有的贡献可以被更可靠的 评估。

\section{2 过滤设计}

为了提高企业的知识吸收能力, 需要建立 过滤机制, 帮助企业更好的评估贡献。数据过 滤可以帮助企业将最有限的资源放在最可能 的贡献上, 提高顾客数据的利用效率。好的过 滤设计识别可靠的贡献, 并对其评估。然而, 不好的过滤机制很容易导致评估偏差, 可能会 导致错误选择决策, 例如可能会导致错过好的 贡献。企业必须精心设计评价机制。例如, AlphaCorp 改善评估过程的质量, 引入一些其 他的中性评级指标, 而不是仅仅评级好或者不 好。

研究表明, 众包人应使用多标准、多维度 的过滤指标 ${ }^{[6]}$, 例如新颖性、相关性和可用性。 这样比单一的评价指标 (如好或者不好) 更准 确。创建可靠的质量排名, 大约需要 20 个评 价指标。另外, 包含相同的内容多个贡献和不 包含任何有价值信息的垃圾邮件, 也会阻碍贡 献的有效评估。这两类数据影响了对最可能实 现贡献的关注和降低过滤机制的有效性。 
Risk Analysis and Crisis Response in Big Data Era (RAC-16)

AlphaCorp 通过使用多步骤的过程, 减少重复 和垃圾邮件。每一个提交的新贡献都要接受查 重检查。顾客参与者输入其贡献的标题, 自动 与现有贡献进行匹配。匹配成功的话, 参与者 被邀请增加他们的解决方案。参与者要确保解 决方案的新颖性和价值。用评定量表对有价值 的贡献进行评估。最后, 不适合的解决方案会 被标记出来。

过滤机制通过选择合适的规则来实现, 合 适的选择规则帮助企业过滤解决方案, 自动的 在成千上万的解决方案中选择可能有价值的 方案。因此, 选择规则规范了企业所能看到的 贡献的类型。例如, BetaCorp 的选择规则提 取极致化的解决方案。这些解决方案也许会有 些争议, 一些企业员工认为其很有价值, 而另 外一些企业员工认为没有价值。但这样的贡献 会引发顾客与顾客展开激烈讨论, 在讨论的过 程中, 参与人可能会提供一些真正有价值的见 解。

\section{3 社区创建}

企业需要的是一个有着众多顾客参与的 社区, 并且该社区有自组织结构。社区需要不 断有新的顾客加入, 创造共同的价值和规范, 特别是信任。一个功能强大的社区能提高吸收 能力。因为企业基本不需要通过资源对平台进 行管理。所有的活动都是以自组织的形式开 展。建立活跃的顾客平台的关键在于吸引大量 的顾客参与 ${ }^{[7]}$ 。平台的参与者越多, 平台的吸 引力就越大。BetaCorp 在其发布的产品上, 增加了反馈的功能, 这可以获得新的参与者。 顾客平台要维系新的参与者, 并刺激他们持续 参与, 参与者会对平台产生感情。为了达到这 个目标, BetaCorp 投入大量资源建立了顾客 社区, 包括创建顾客认同的共享文化。

通过建立自组织结构, 可以加强情感联系, 促进参与者积极主动的参与社区的管理。这种 情感联系可以培养参与者之间, 参与者与企业 员工之间的社会联系。这种联系有助于信息交 换、数据评估和传播。例如, AlphaCorp 和 GammaCorp 为参与者定义特定的角色和权利。 每个层级的参与者允许执行不同的活动, 越活
跃, 越得到承认的参与者, 级别就越高。增加 顾客参与者的授权, 可以高度激励顾客参与 者, 有利于增强参与者的情感联系。

\section{Acknowledgements}

This study was supported by the State Ethnic Affairs Commission Ethnic problem research project (2015-GM-076).

致谢

本研究得到了 2015 年国家民委民族问题 研究项目 [2015-GM-076] 的资助。

\section{参考文献:}

[1]常静,杨建梅,欧瑞秋.大众生产者的参与动 机研究述评.科技管理研究，2009， (5):423-425.

[2]杰夫.豪.众包一一大众力量缘何推动商业未 来.牛文静译.北京: 中信出版社, 2009.

[3]胡晓琼.众包: 新形势下低成本揽才新思路. 中国人力资源开发,2010,238(4): 25-27.

[4]H.Chesbrough. Open innovation, the new imperative for creating and profit from technology. Harvard business school press, 描述 2003.

[5]刘文华,阮值华.众包-让消费者参与创新. 企 业管理,2009,(7): 93-95.

[6]魏栓成,邬适融.众包的产生、发展以及构建 众包商业模式应遵循的路径. 上海管理 科学,2010, 32(1): 55-58.

[7]刘宏.电子商务中威客盈利模式探讨. 商业 时代,2008,(5):92.

[8]付敬,朱桂龙.知识源化战略、吸收能力对企 业创新绩效产出的影响研究. 科研管理, 2014, 35(3):25-34.

[9]F.S. Yu, S.H. Liu. Decision-making model in the environment of complex structure data. Journal of Risk Analysis and Crisis Response, 2013,3(2):103-109. 